\title{
Bei begründetem Verdacht auf atypische Pneumonie gleich dual behandeln!
}

Im Wahlkampf um die US-Präsidentschaft hatte die atypische Pneumonie von Hillary Clinton die Frage nach ihrer gesundheitlichen Eignung aufgeworfen. Ist eine atypische Pneumonie denn ein Indikator für eine insgesamt angegriffene Gesundheit?

Oesterlee: Sie kann ein Hinweis auf eine angegriffene Gesundheit sein, muss aber nicht. Im Prinzip kann jeder eine atypische Pneumonie bekommen. Bei älteren Menschen wie Hillary Clinton kommen als Erreger einer Pneumonie neben Pneumokokken häufiger Enterobakterien infrage und nicht die atypischen Erreger.

Das heißt, jüngere Menschen sind stärker gefährdet, an einer atypischen Pneumonie zu erkranken?

Oesterlee: Atypische Erreger wie zum Beispiel Mykoplasmen treten bei jüngeren Menschen häufiger als Pneumonieerreger auf als bei älteren Menschen. Im Alter bewirken zudem Veränderungen der oropharyngealen Flora und Komorbiditäten, dass häufiger auch gramnegative Erreger wie zum Beispiel Enterobakterien eine Pneumonie verursachen.

Lässt sich abschätzen, wie hoch unter den Pneumonien der Anteil mit atypischen Erregern ist?

Oesterlee: Streptococcus pneumoniae ist mit 40-50\% immer noch mit Abstand der häufigste Erreger der ambulant erworbenen Pneumonie. Dann gibt es eine Gruppe von selteneren Erregern mit einer Häufigkeit von 5-10\%. Dazu zählen neben Haemophilus influenzae auch atypische Erreger wie Mykoplasmen und respiratorische Viren wie RS-Viren, Influenzaviren oder Adenoviren. In weniger als 5\% der ambulant erworbenen Pneumonien findet man als Erreger neben Staphylokokken auch Legionellen und Chlamydien. Man geht aber davon aus, dass Pneumonien mit atypischen Erregern häufiger sind als bisher angenommen. Wahrscheinlich sind vor allem Erkrankungen mit leichtem Verlauf unterdiagnostiziert.

Wann sollte der Arzt Verdacht auf eine atypische Pneumonie schöpfen?

Oesterlee: Die Definition der atypischen Pneumonien ist unscharf. Zum einen ist sie für atypische Erreger - Chlamydien, Legionellen, Mykoplasmen und respiratorische Viren - im Gebrauch. Zum anderen wird sie auch für eine atypische Klinik verwendet. Dementsprechend ist bei schleichendem Krankheitsbeginn und Krankheitsverlauf mit wenig Fieber und kaum oder keinem Auswurf sowie atypischen Infiltraten im Röntgenbild an eine atypische Pneumonie zu denken. Beschwerden, die nicht unmittelbar die Atmungsorgane betreffen, wie Kopfschmerzen, Muskelschmerzen und Diarrhoe, sowie Laborauffälligkeiten wie Hyponatriämie, Transaminasenerhöhung und Erhöhung der Retentionsparameter sollten auch an eine atypische Pneumonie denken lassen. Wichtig ist, dass man, wenn man unter Verdacht auf eine Pneumonie behandelt, egal ob sta-

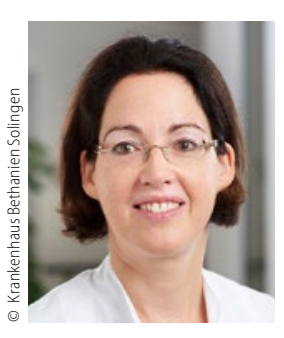

Ulrike Oesterlee

Oberärztin der Klinik für Pneumologie und

Allergologie, Krankenhaus Bethanien, Solingen

tionär oder ambulant, den Patienten immer ein zweites Mal sieht. Dafür wird ein Intervall von 72 Stunden nach Therapiebeginn empfohlen. Wenn der Patient dann kein Ansprechen auf die Antibiose zeigt, muss man auch an atypische Erreger denken.

\section{Wie geht man dann vor?}

Oesterlee: Ganz wichtig ist, Anamnese und Diagnostik wie CRP und Röntgen zu wiederholen und gegebenenfalls die Diagnostik auf atypische Erreger zu ergänzen. Zur Anamnese gehört auch die Frage, ob der Patient verreist war und ob auch Personen aus dem Umfeld erkrankt sind. Man sieht sich die Vitalparameter nochmal genau an - Sauerstoffsättigung, Atemfrequenz, Herzfrequenz, Blutdruck, Temperatur, Bewusstseinsstatus. Wenn ein ambulanter Patient sich nach 72 Stunden trotz Antibiose verschlechtert hat, wäre meine Empfehlung, ihn einzuweisen.

Wie unterscheidet sich die Therapie bei atypischer bakterieller Pneumonie von der bei typischer Pneumonie?

Oesterlee: Zur Therapie der ambulant erworbenen Pneumonie werden Betalaktamantibiotika empfohlen, eventuell zusammen mit einem Betalaktamaseinhibitor. Das Problem ist, dass diese Medikamente nicht auf atypische Erreger wirken. Das heißt, man muss bei Verdacht oder Nachweis eines atypischen Erregers die Therapie direkt anders beginnen, wechseln oder um ein Atypen-wirksames Antibiotikum erweitern, zum Beispiel ein Fluorchinolon wie Levofloxacin oder Moxifloxacin oder ein Makrolid wie Clarithromycin oder Azithromycin. Es ist ratsam, bei schwerer Erkrankung direkt dual zu beginnen. Man soll zwar eine Diagnostik auf atypische Erreger machen, aber die dauert zum Teil recht lang und ist oft auch nicht verlässlich. Deswegen ist es besser, bei berechtigtem Verdacht auf einen atypischen Erreger die Therapie frühzeitig zu modifizieren.

Das Interview führte Dr. Beate Schumacher 